\title{
Analysis of Effect of Symmetric Tiles on Binary Counter in DNA Self-Assembly
}

\author{
Aijun Zhu ${ }^{1,2, *}$, Duanyong Chen ${ }^{1,2}$, Cong $\mathrm{Hu}^{1,2}$, Chuanpei $\mathrm{Xu}^{1,2}$,Aiguo Song ${ }^{3}$
}

1. School of Electronic Engineering and Automation, Guilin University of Electronic Technology, Guilin ,China;

2. Guangxi Key Laboratory of Automatic Detecting Technology and Instruments, Guilin, China;

3. School of Instrument Science and Engineering, Southeast University, Nanjing, China

*45953000@163.com

Keywords: Symmetry tile; DNA Self-Assembly; Counting

\begin{abstract}
With the growth of complicate system or crystals, whereas, a few phenomena have occurred and been found, which could result in final crystals or patterns with errors. Among those phenomena, one has still not been processed in current published literatures, which is named symmetry tile may appear. This paper analyses the effect of symmetry tile on binary counter in DNA Self-Assembly. The exact relationship between symmetry tile and counting is set up considering the type of symmetry and the position of the symmetry tile. The analysis shows that the final patterns are partly or completely different from the originally desired patterns in the case of symmetry tile due to the changed counting behavior. Simulation results which are obtained by Xgrow demonstrate that the analysis is valid and correct.
\end{abstract}

\section{Introduction}

As we all know, Self-Assembly is commonly regarded as an emerging technology which could be used in nano-fabrication or computation because of its nanoscale feature size [1, 7-11]. In nano-manufacture system and algorithmic system, DNA is applied in Self-Assembly as a main technology because of its wonderful versatility. From the bottom to up, DNA offers a skeleton frame to dispose small and standard blocks such as tiles, which can grow and become a complicate system or pattern. Without lose of generality, DNA Self-Assembly grows from a seed tile and continues its growth by addition of boundary tiles and rule tiles along a special direction. Usually, it grows from the southeast corner to northwest with the current aggregate. The attachment of tiles is according to a strict rule with the growth corner and bonding characteristic. With the growth of complicate system or crystals, whereas, a few phenomena have occurred and been found, which could result in final crystals or patterns with errors [2]. Among those phenomena, one has still not been processed in current published literatures, which is named symmetry tile probably appear [3]. In such case, some bonding behaviors have been changed compared with the original tile set. In the case of symmetry tile, the final patterns are partly or completely different from the originally desired patterns; this is because so far as to the hypothesis of no match error, the corresponding growth would probably carry through with unpredictable final patterns.

In self-assembly manufacture system and algorithmic application, counting is a general operation for self-assembly and also an elementary step. The binary counter and its corresponding pattern are distinguished tile set and graphic pattern. For example, the binary counter is adopted as one fundamental part and component of a demultiplexer in literature. M.Y.Kao adopted binary counter to assemble a square [4-6]. M.Hashempour gave the analysis of effects of rotated tile on binary counter [7]. This paper mainly discusses the effect of symmetric tile on counting procedure.

Organization of the rest of this paper is as follows. The mathematical model is briefly introduced in Section 2. Tile symmetry effects on counting are presented in Section 3. Counting rules after single tile symmetry is introduced in Section 4. Finally, concluding remarks is given in Section 5. 


\section{Mathematical Mode}

To set up the model of the growth procedure in DNA self-assembly, it is very convenient to adopt a transition graph for making sure the attachment in a certain growth corner. The transition graph is defined as a directed graph $P(D, B)$, where $D$ is a vertex set and $B$ is a directed edge set. As direction of the growth starts from south-east to north-west, the south-east corner is defined as the growth corner. The directed edge set B is the whole feasible attachments in all growth corners. Therefore, every rule tile is consisted of two nodes and a directed edge, which is expressed Fig. 1 as follows.

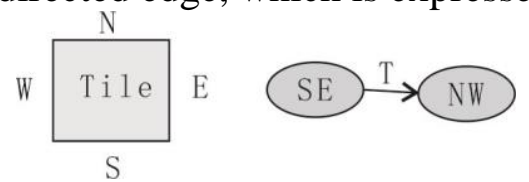

Fig.1 Tile (left) and its transition graph (right)

In this way, every tile set has its specific transition graph P, and in Fig.2 the tile set of binary counter pattern is demonstrated as follows.

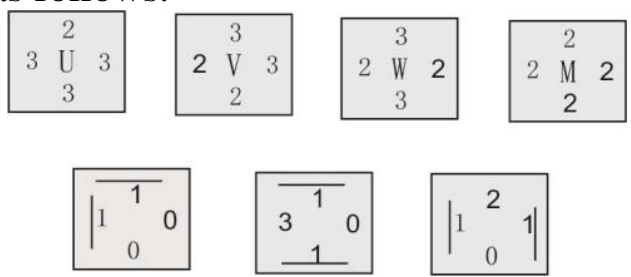

Fig.2 all the tiles in the binary counter tile set

Because contribution of growth is mainly achieved by rule tiles, we only show the transition graph for the rule tiles of binary counter pattern in Fig.3. In binary counter pattern, there is a seed tile $[(\mathrm{N}, \mathrm{E}, \mathrm{S}, \mathrm{W})][(1,0,0,1)]$ and two boundary tiles $[(2,1,0,1),(1,0,1,3)]$. Rule tiles $[(2,3,3,3),(3,3,2,2)$, $(3,2,3,2),(2,2,2,2)]$ are named as $[\mathrm{U}, \mathrm{V}, \mathrm{W}, \mathrm{M}]$, respectively. In the case of counting, $\mathrm{V}$ and $\mathrm{W}$ denotes digit " 1 ", and the corresponding $\mathrm{U}$ and $\mathrm{M}$ denotes digit " 0 ". If the tile set of binary counter attach together with zero error rate, the counter number with increase one by each line from zero.

Suppose P'(D',B') be symmetric transition graph which is achieved by the symmetry of the original rule tiles. Suppose $\mathrm{P}(\mathrm{D}, \mathrm{B})$ is the original transition graph. $\mathrm{X}$ axis symmetry of rule tile is defined as the exchange of North with South. Y axis symmetry of rule tile is defined as the exchange of West with East. XY axis symmetry of rule tile is defined as both the exchange of North with South and the exchange of West with East. Therefore, the following situations should be taken into account as to the relationship between $\mathrm{P}$ and $\mathrm{P}^{\prime}$.

(1) $\mathrm{P}^{\prime}=\mathrm{P}$, which also means $\mathrm{D}^{\prime}=\mathrm{D}$ and $\mathrm{B}^{\prime}=\mathrm{B}$. In this case, the symmetric tile is exactly the same as the original tile. In the tile set of binary counter, there are rule tiles $\mathrm{W}$ and $\mathrm{M}$ which belong to such case. That means all symmetries (X, Y, or XY) of tile $\mathrm{W}$ or $\mathrm{M}$ will lead to the identical tile.

(2) $\mathrm{P}^{\prime} !=\mathrm{P}$, to be exact, $\mathrm{D}^{\prime}=\mathrm{D}$ and $B^{\prime}=B \cup\{\forall b \mid b \notin B\}$.In this case, there are no new vertexes but new edges. It means that it does not create new growth corner. There are still two subcases which can be made a distinction between. The first subcase is that the new edge links two existing vertexes which are concatenated too in P. For example, a XY axis symmetry of rule tile V will result in a totally new edge between nodes [3, 2] and [2,3] in P'. Consequently, transitions between nodes are provided with different choice and not unique. Naturally, the final assembled shape may be totally dissimilar with the wanted one, even under the assumption of no mismatch. The second subcase is that the new edge does not link two existing vertexes in P. For example, $\mathrm{X}$ axis symmetry of rule tile $\mathrm{V}$ will result in a totally new edge between nodes $[3,3]$ and $[2,2]$ in P'. As a result, transitions between the two nodes are not unique. Naturally, the final assembled shape may be totally dissimilar with the expected one, even under the assumption of no mismatch.

(3) $\mathrm{P}^{\prime} !=\mathrm{P}$, to be exact, $D^{\prime}=D \cup\{\forall d \mid d \notin D\}$ and $B^{\prime}=B$. Such a case is impossible theoretically. Because according to the definition of $\mathrm{P}$, if there is a new node, then the node must be provided with an out-edge. Therefore, $B^{\prime}=B$ is impossible and invalid. 
(4) $\mathrm{P}^{\prime} !=\mathrm{P}$, to be exact, $D^{\prime}=D \cup\{\forall d \mid d \notin D\}$ and $B^{\prime}=B \cup\{\forall b \mid b \notin B\}$. This case happens in most situations. There are also two subcases probably. Case one: the new nodes in the symmetric transaction graph may be connected to the other nodes in P. As a result, new edges are created and the transitions between certain nodes are not unique. Consequently, it is possible to result in a final assembly which is not desired. Case two: the new nodes in the symmetric transaction graph are not connected to the other nodes in P. In such case, it is not possible to obtain a new growth corner for the attachment of symmetric tile. Consequently, under the assumption of no mismatch, the final assembly is just the desired pattern.

\section{Tile Symmetry Effections on Counting}

The circumstances and example of section two could be discussed by considering several counting sequence in binary counter pattern. The symmetric transition graph $\mathrm{P}^{\prime}$ of the binary counter tile set is demonstrated in Fig.3 as follows, which is the symmetric transition graph for all symmetric tiles except those are the same, such as $\mathrm{W}$ and $\mathrm{Wx}$ or M and Mxy.

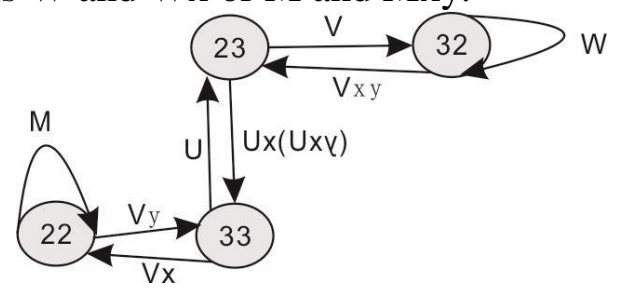

Fig.3 symmetry transition graph of binary counter tile set

The symmetry effects on counting are evaluated under the assumption of at most one symmetric tile in every row. Consequently, the symmetry effects on counting could be obtained from the above mathematical model. For instance, Uy,Wx, Wy, Wxy, Mx, My and Mxy would not lead to new tile and their corresponding symmetric graph is exactly the same as the original one. Nonetheless, $\mathrm{Ux}, \mathrm{Uxy}, \mathrm{Vx}, \mathrm{Vy}$ and $\mathrm{Vxy}$ would lead to new rule tile with an new edge in $\mathrm{P}^{\prime}$ for every symmetry. With no doubt, $\mathrm{P}$ is sure to change with respect to symmetry. The exact effects of all symmetry are shown in TABLE 1.

TABLE 1 TILE SYMMETRY EFFECTS ON THE SYMMETRIC TRANSITION GRAPH
\begin{tabular}{|l|l|l|l|l|l|l|l|l|}
\hline & $\mathrm{U}_{\mathrm{X}}$ & $\mathrm{U}_{\mathrm{Y}}$ & $\mathrm{U}_{\mathrm{XY}}$ & $\mathrm{V}_{\mathrm{X}}$ & $\mathrm{V}_{\mathrm{Y}}$ & $\mathrm{V}_{\mathrm{XY}}$ & $\mathrm{W}_{\text {All }}$ & M $_{\text {All }}$ \\
\hline New node & No & No & No & No & No & No & No & No \\
\hline New edge & Yes & No & Yes & No & Yes & Yes & No & No \\
\hline Added tile & $(3,3,2,3)$ & - & $(3,3,2,3)$ & $(2,3,3,2)$ & $(3,2,2,3)$ & $(2,2,3,3)$ & - & - \\
\hline Added edge & $23 \rightarrow 33$ & - & $23 \rightarrow 33$ & $33 \rightarrow 22$ & $22 \rightarrow 33$ & $32 \rightarrow 23$ & - & - \\
\hline
\end{tabular}

By the tile set of Fig.2, the counter with no mismatch begins to count from zero in increasing by one. Each row in the pattern means a count. Suppose a tile be denoted by a four-tuple (N, E, S, W), which indicate the values of the bonds at north, east, south and west, respectively. Under the assumption of no mismatch and no symmetry, there are sure to be some relations between the tile sequence and the counting digital. Let the tile $(3,3,2,2)$ and the tile $(3,2,3,2)$ denote the equivalence of a digital value " 1 ", and let the tile $(2,2,2,2)$ and the tile $(2,3,3,3)$ denote the equivalence of a digital value " 0 ". Consequently, symmetry may lead to a tile denoting a " 1 " substituted by a tile denoting a "0". There are also other changes which may occur. For instance, an X symmetry in tile $(3,3,2,2)$ (a digital " 1 ") would lead to tile $(2,3,3,2)$. That is just similar with a new tile and according to its growth corner (east and south), it will attach at the place of tile $(2,3,3,3)$ (a digital "0") in the final assembly. Such an example means a symmetry tile can substitute a different tile with a distinct value, which definitely would change the corresponding counting sequence.

The following section will discuss several examples at length in order to demonstrate different situations where a symmetry tile would impact the real counting procedure in the final assembly pattern. 


\section{Rules after a Single Tile Symmetry}

With the previous analysis, a detailed discussion of binary counter with single tile symmetry is as follows. To simplify the analysis, we assume that there is only one single tile symmetry on every row. As we know, every row in the binary counter can be classified into four sections. The value of the counter with the tile symmetry is analyzed as follows. Due to the tile symmetry, the value of the counter is not $\mathrm{N}+1$ but getting a different value and the counting sequence would begin counting from such a value. Let $\mathrm{j}$ is the exact position of the symmetry tile. Furthermore, the distinct effects in every region of a row are demonstrated too. The string "M..MW..WM..MW..WVU..U" means a row of a binary counter. The right is the least significant digit and the left is the most significant digit. There are either zero "U" or more (such region is called "U" region), exactly one "V",either zero "W" or more , and either zero "M" or more. These include all feasible cases which might occur in binary counter which is achieved by self-assembly.

The dissimilarity between the counter value before (shown by $\mathrm{N}$ ) and after a single tile symmetry (shown by $\mathrm{N}_{\text {next }}$ ) is discussed as follows. As to each case of single tile symmetry, a strict proof is provided. There are several possible cases as follows.

1) Any Vx symmetry: Next is in a boundary between $N$ (called delay mode) and 1 (called reset mode).

Firstly, consider such a case that Vx will affect the "U" region which are located in the least significant zeros and on the right side of the least significant ones. Such $\mathrm{N}_{\text {next }}$ is at the right side. Let $\mathrm{j}$ equal 0 then $\mathrm{N}_{\text {next }}=\mathrm{N}$.

Secondly, suppose the symmetry affect the most significant zero of the "U" region. Let the "U" region are $t$ bits long, then we obtain

$$
\begin{aligned}
& \mathbf{N}=\mathbf{2}^{t}-1+\text { constant } \text { and } j=t-1, \\
& \mathbf{N}_{\text {next }}=\mathbf{2}^{t}-1+\text { constant }-\mathbf{2}^{t-1+1}+2=\text { constant }+1,
\end{aligned}
$$

2) Any Ux or Uxy symmetry: $\mathrm{N}_{\text {next }}$ is in a boundary between $\mathrm{N}+4$ and $3 * N+4$.

Proof: these symmetry tiles will influence " $\mathrm{V}$ " region evidently as their growth corners are the same as that of "V" region. Firstly, as "V" region may be the least significant bit while $\mathrm{j}$ equals zero, then $\mathbf{N}_{\text {next }}=N+\mathbf{2}^{0+1}+2=N+4$, which is its lower boundary.

Secondly, suppose "V" region is the most significant bit of binary counter. Let the binary counter are $\mathrm{t}$ bits long, then we obtain

$$
\begin{aligned}
& \mathbf{N}=\mathbf{2}^{t}-1+\text { constant } \text { and } j=t, \\
& \mathbf{N}_{\text {next }}=N+\mathbf{2}^{t+1}+2 \leq 3 \times N+4
\end{aligned}
$$

Simulation results which are obtained by Xgrow demonstrate that the above analysis is valid and correct. Parts of the simulation results are demonstrated in Figs 4.

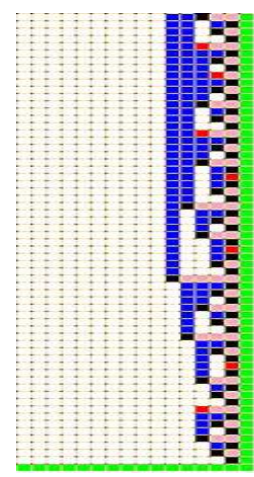

Fig.4 Vx symmetry and its impact on binary counter 


\section{Conclusions}

This paper provides detailed analyses with the effect of symmetry tile on binary counter in DNA Self-Assembly. The exact relationship between symmetry tile and counting is set up considering the type of symmetry and the position of the symmetry tile. The analysis shows that the final patterns are partly or completely different from the originally desired patterns in the case of symmetry tile due to the changed counting behavior. Simulation results which are obtained by Xgrow demonstrate that the analysis is valid and correct.

\section{Acknowledgment}

This research has been partially funded by National Natural Science Foundation of China (61561012, 61562013), Guangxi Key Laboratory of Automatic Detecting Technology and Instruments (YQ16110), Talent Project of Guilin university of electronic technology (UF15008Y), Guangxi Natural Science Foundation (2017GXNSFAA198021), China Scholarship Council (201508455020).

\section{References}

[1] S. Zhang, D. M. Marini, W. Huang, and S. Santoro, "Design of nanostruc-Tured biological materials through self-assembly of peptides and proteins,"Curr. Opin. Chem. Biol., vol. 6, pp. 865-871, 2002.

[2] M. Cook, P. W. K. Rothemund, and E. Winfree, "Self-assembled circuitpatterns," DNA Computers 9, (Lecture Notes in Computing Science,vol. 2943). New York: Springer-Verlag, pp. 91-107, 2004..

[3] F. Fujibayashi and S. Murata, "Precise simulation model for DNA tile self-assembly," IEEE Trans. Nanotechnol., vol. 8, no. 3, pp. 361-368,May 2009.

[4] M. Hashempour, Z. M. Arani, and F. Lombardi, "A graph model for tile sets in DNA self-assembly," in Proc. IEEE Int. Workshop Design Test Nano Devices, Circuits Syst., Boston, MA, Sep. 2008, pp. 77-81. (Lecture Notes in Computing Science,vol. 2943). New York: Springer-Verlag, pp. 91-107, 2004..

[5] M.Hashempour,Z.M.Arani,andF.Lombardi,"Healingassessmentoftile sets for error tolerance in DNA self-assembly," IET Trans. Nanobiotech-nol., vol. 2, no. 4, pp. 81-92, Dec. 2008.

[6] V.S. Kulala, S. S. Kanangat, and J. Joshy, "Fullerene Cluster Assisted Self-Assembly of Short DNA Strands into Semiconducting Nanowires," CHEMISTRY-A EUROPEAN JOURNAL., vol. 23, no. 62, pp. 15759-15765, Nov 2017.

[7] M. Hashempour, Z. M. Arani, and F. Lombardi, "Counting by DNA Self-Assembly in the Presence of Rotated Tiles,” IEEE Trans. Nanotechnol., vol. 10, no. 3, pp. 77-81, May 2011.

[8] C. Fiona W, J. M. Alexander, T Andre, "Time lapse microscopy of temperature control during self-assembly of 3D DNA crystals," JOURNAL OF CRYSTAL GROWTH., vol. 476, no. 3, pp. 1-5, Oct 2017.

[9] Jiang, Shuoxing; Hong, Fan; Hu, Huiyu, "Understanding the Elementary Steps in DNA Tile-Based Self-Assembly,” ACS NANO, vol. 11, no. 9, pp. 9370-9381, Sep 2017.

[10] Sun, Dandan; Guo, Tuan; Guan, Bai-Ou, "Counting by DNA Self-Assembly in the Presence of Rotated Tiles," JOURNAL OF LIGHTWAVE TECHNOLOGY., vol. 35, no. 16, pp. 3354-3359 , Aug 2017. 
[11] Mohammed, A. M.; Velazquez, L.; Chisenhall, A.; "Self-assembly of precisely defined DNA nanotube superstructures using DNA origami seeds," NANOSCALE, vol. 9, no. 2, pp. 522-526, Jan 2017. 